\title{
Semantic Approaches to Structuring and Querying Web Sites
}

\author{
E. Damiani \\ Università di Milano - Polo di Crema \\ Via Bramante, 65, I-26013 Crema, Italy, edamiani@crema.unimi.it \\ L. Tanca \\ Università di Verona and Politecnico di Milano \\ Ca'Vignal 2, I-37134 Verona, Italy, tanca@sci.univr.it
}

\begin{abstract}
In order to pose effective queries to Web sites, some form of site data model must be implicitly or explicitly shared by users. Many approaches try to compensate for the lack of such a common model by considering the hypertextual structure of Web sites; unfortunately, this structure has usually little to do with data semantics. In this paper a different technique is proposed that allows for both navigational and logical/conceptual description of Web sites. The data model is based on $W G$-log, a query language based on the graphoriented database model of GOOD (Gyssens et al. 1997) and G-log (Paredaens et al. 1995), which allows the description of data manipulation primitives via (sets of) graph(s). The WG-log description of a Web site schema is lexically based on standard hypermedia design languages, thus allowing for easy schema generation by current hypermedia authoring environments. The use of $W G-\log$ for queries allows graphic query construction with respect to both the navigational and the logical parts of schemata. Site schemata are managed by Schema Robots, which assist clients in the process of identification and retrieval of a set of candidate schemata. On the basis of the set of candidate schemata, the client may then query individual Web sites; extensive data caching is used to avoid flooding resulting from an excessive number of candidates. A remote Query Manager process, running side by side with standard Web servers, manages query execution and handles the presentation of the results to the client. Our schema is particularly suited for Intranets, while allowing for a smooth migration of Internet Web sites as more and more of them are produced on the basis of hypermedia design and generation methodologies.
\end{abstract}

\section{Keywords}

World Wide Web, query languages, schema-based semantics of Web sites 


\section{INTRODUCTION}

The steady growth in the amount of data published via the World Wide Web (WWW) has led to a number of attempts to index Web's contents. Initially, these attempts only tried to collect and index title-like information about every reachable page of data on the WWW, and then build Boolean keyword searches into that database. Today, one could hardly find a Web search engine relying on term indexing alone. However, although many of the new search engines present a sophisticated query interface, the results they deliver are perceived by the user community as unsatisfactory. In our opinion, this situation is mainly due to the following three causes:

\section{- Noise effect}

In the current systems, query language interfaces suggest precise semantics while the underlying keyword search mechanism remains mainly syntactical in nature and therefore prone to the well-known noise and silence sideeffects. Sophisticated indexing techniques exploiting HTML tagging may relieve this problem, but by no means solve it completely.

- Flatness of results Currently, query results are flat lists of pages that do not capture the underlying structure of the searched Web sites. As a consequence, retrieved information neither is easily presented to the user nor can be efficiently reorganized.

- Non-HTML objects indexing The increasing presence of non-HTML objects (Hu et al. 1996) attached to Web pages (such as multimedia clips or Java applets) jeopardizes automatic index construction and update.

The above considerations suggest to complement keyword-based searching with database-style support for querying the Web. Several research projects addressed this problem, and are reviewed in Section 2

\subsection{An outline of our approach}

Our approach, differently from the others, is based on the following two principles:

- The availability of a schema is a prerequisite for the development of an effective query mechanism, since the schema carries most of the semantic information needed for querying.

- If a well specified methodology is used to design a Web site, some notion of schema is present during the site design process 
Logical Description Languages

(e.g. G-Log, GOOD)

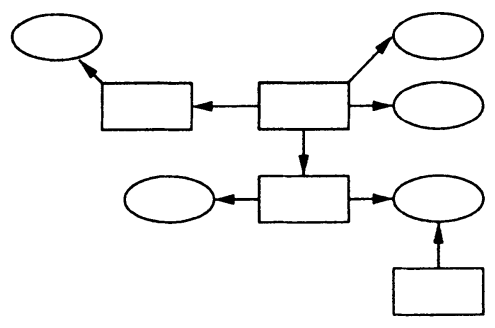

Mixed logical/navigational description languages (Automatic publishing tools e.g. MS FrontPage)

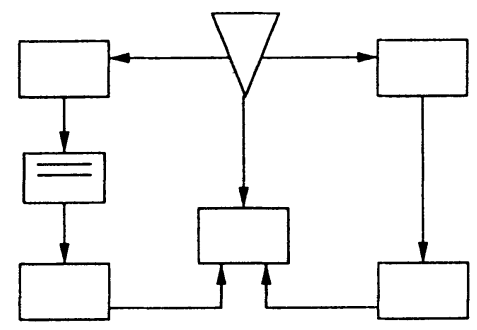

Real Web Pages (static or dynamic)

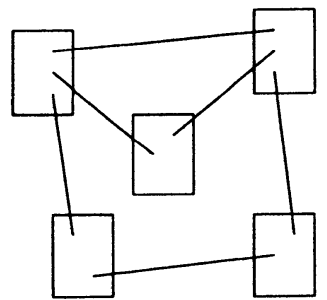

Figure 1 Links between conceptual and hypermedia description languages

So, this paper proposes to reuse site design artefacts to attain (semi)automatic construction of schemata for Web sites. As outlined in Fig. 1, many popular authoring environments for Web sites already hint at the idea of some sort of navigational schema to be chosen by the user as the basis of automatic site generation. Moreover, several research prototypes of Web site generators (Fraternali et al. 1997)) based on hypermedia design languages such as HDM (Garzotto et al. 1995) (Hypermedia Design Method), YOO (Balasubramanian et al. 1995), RMM (Isakowitz et al. 1995) and the like, are becoming available. This suggests the possibility of semi or fully automatic generation of schemata during the design process, allowing for smooth migration to a more organized Web as more and more sites are produced on the basis of standard design and generation methodologies. In our approach, Web site schemata form a distributed hierarchy, managed by server processes called Schema Robots. A Schema Robot is a server process which mantains and provides information 
about Web sites schemata, stored and presented in $W G$-log form, to the clients in execution over the Net. Although in this paper we will not elaborate on site classification issues, it is worthwhile to remark that Schema Robots need not be all equal; for instance, a set of domain schema robots might form a distributed partitioned repository of all known schemata on the Net, while category schema robots might allow for a subject-related search. In this perspective, Robots can also be regarded as browsable similarity-based hypertexts of WG-log schemata, providing semantically rich information about Web sites. This hypertextual structure should not be regarded simply as "another technique" for schema identification; the availability of large, searchable schema repositories may prove a significant contribution to the much needed Web restructuring via resource indexing systems (Budi et al. 1996). All queries

are formulated w.r.t. a site schema, supposed to be known in advance to the client formulating the query. In order to formulate and execute the query, the following steps are performed:

1. Schema identification

2. Schema retrieval and query formulation

3. Instance retrieval

4. Presentation of results

In the following, we shall describe each step in some detail.

\section{- Schema identification}

To get the best results, queries to the Web must be formulated on the basis of a known schema. In order to help the user in identifying a suitable schema w.r.t. the planned query, facilities for schema browsing and keyword-searching, together with a Thesaurus are available at Robot sites. The Thesaurus is mainly intended to provide a browsable controlled vocabulary to help the user in getting acquainted with the schema data dictionary. Note that, as the number of stored schemata will grow, automatic Thesaurus inizialization and update (Damiani et al. 1995) will become crucial for the Robots' performance.

- Schema retrieval

With the help of the keyword-based information provided by the user, the Schema Robot's search engine identifies candidate schemata to be proposed to the user. With the help of a graphical editor, the user constructs appropriate queries for the schemata identified by the Robot; therewith, on reception of the'schema-based user queries, the Robot consults an instance cache to discard sites that surely do not contain the desired information. This cache holds, for each schema, the values of selected attributes of some entities; aging mechanisms are required to ensure its consistency. The result of this second step is a list of Web sites together with valid references to them, i.e. the network addresses of the associated query manager processes. 


\section{INTRODUCTION}

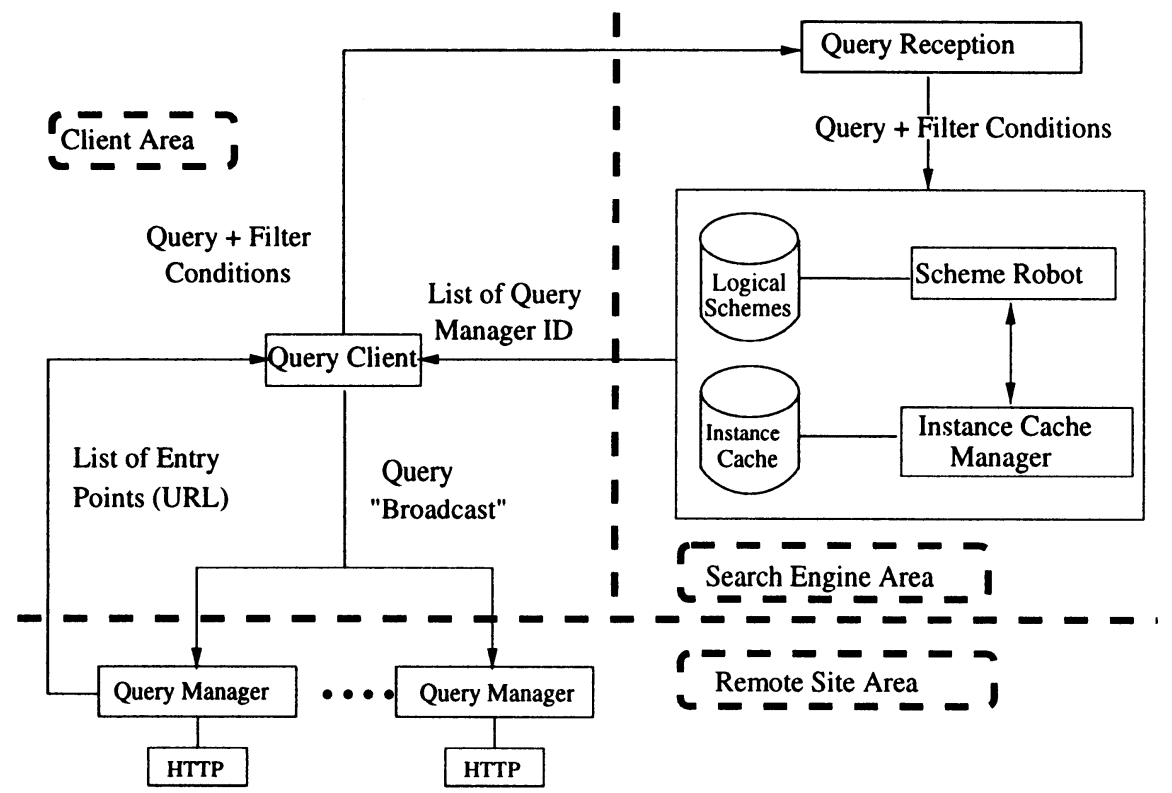

Figure 2 System architecture

\section{- Instance retrieval}

The query is now broadcast to all query managers running on candidate Web sites. If the candidate Web site is based on an underlying database, the Query Manager process can provide an interface to the existing DBMS. In general, however, the Query Manager will maintain a copy of the WGlog schema and some indexing information linking navigational entities to (a list of) URLs. The resulting instance is computed at the target site; efficiency is critical at this moment, both in terms of time of computation of the resulting instance and of amount of gathered data that has to be trasmitted to the originating site.

\section{- Presentation of Results}

This last step performs gathering and presentation of the result pages, on the basis of the data structures mapping HTML pages and the navigational part of the schema. This is a very interesting problem, since the amount of information contained in the resulting instance may be huge and the user must be presented with a synthesis of the available information, organized in such a way that he/she can choose to browse the resulting instance according to different perspectives. The synthetical result submitted to the user must enable him/her to formulate one or more appropriate goals that select exactly the needed information from the computed instance, to be transported to the originating site.

Fig.2 summarizes the system architecture. This paper is organized as follows: 


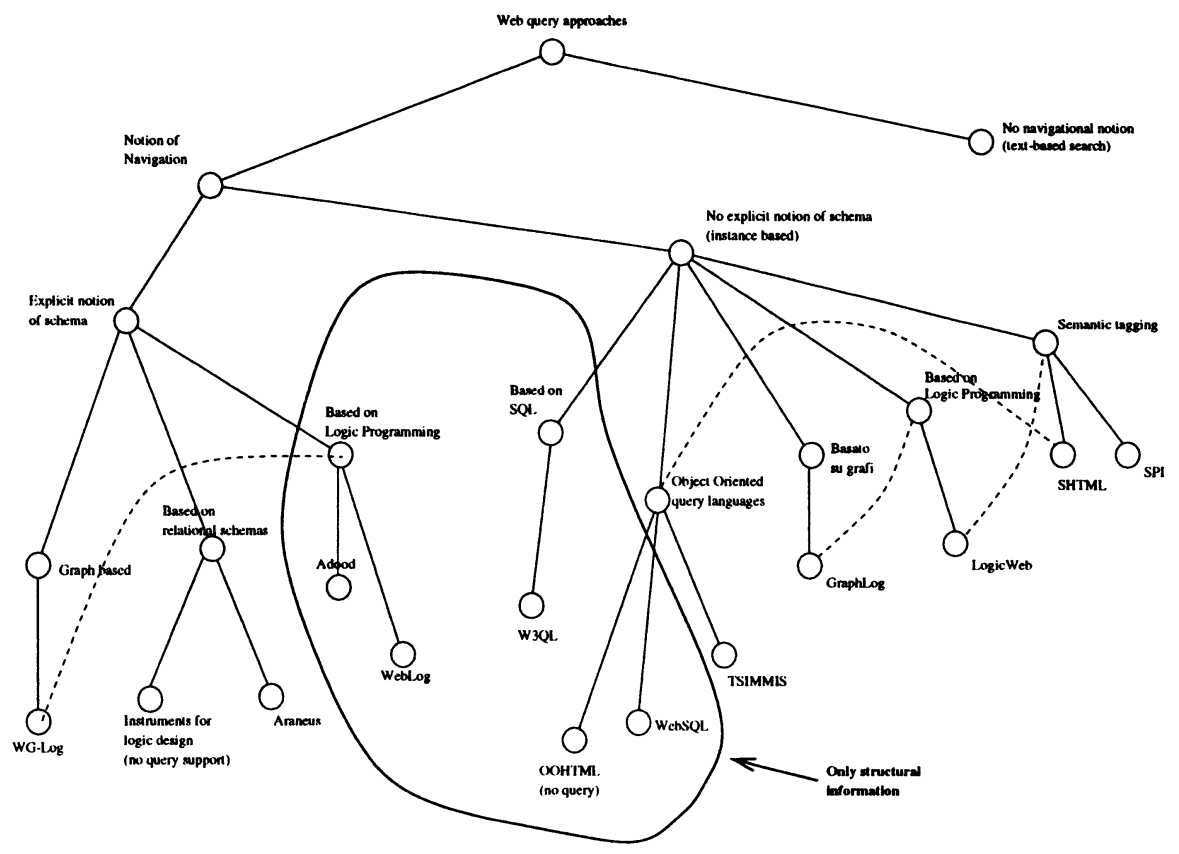

Figure 3 A semantics-based classification of research approaches to WWW querying

Section 2 presents a survey of existing techniques, Sections 3 and 4 describe the data model and language of WG-log; Section 5 presents the naive computation of WG-log queries, while Section 6 draws the conclusions and outlines future research on the same subject.

\section{RELATED WORK}

The huge amount of data published via the World Wide Web has led to a number of research efforts on techiques to index, query and restructure WWW sites contents. In this section we provide a brief overview of related work (see also (Torlone 1996)). Our discussion of previous work is based on how the various approaches deal with semantics representation. Figure 3 summarizes the overview.

- Free text indexing - No semantics representation Early approaches to Web indexing tried to collect and index title-like information about every reachable page of data on the WWW and then build Boolean keyword searches into the resulting document. The JumpStation (Fletcher 1990), probably the first well-known system designed to index WWW information, collected 
only information marked by HTML <TITLE>-tags from pages it encountered. Many current Web search engines are still partially based on this approach, where search results are flat lists of HTML pages, completely unrelated to the hypertextual structure of the sites they come from. In the past few years, as the amount of WWW data continued to increase, users grew unsatisfied with pure keyword matching. Nowadays, one could hardly find a Web search engine relying on terms indexing alone. Some keywordbased indexes, like the World Wide Web Worm (WWWW) (McBryan et al. 1994), the WebCrawler (Pinkerton 1997) and Lycos (Lycos 1997) try to complement keyword indexing by taking into account the HTML document structure in order to make educated guesses about semantics. For example, Lycos summarizes the actual content of documents by taking advantage of human-tagged information (HTML headings), of often-appearing keywords and of the introductory text that generally is positioned at the beginning of a file.

\section{- Semantics representation via taxonomies}

Several search engines do not use keyword indexing but exploit a taxonomy representing sites' content. The popular Yahoo (Yahoo! 1997) search engine relies on a broad hierarchical classification systems of subjects, much similar to those used by the Library of Congress or by the ACM Classification of Computer Science Topics. Yahoo's success has spawned multiple similar tools, all based on the idea of providing large, monolithic servers holding indexes of site contents (Point (Lycos-2 1997), Magellan (McKinley 1997), and others). Recently, evolvable taxonomies were proposed, such as the one developed by the CommerceNet Consortium (Hamilton 1997). In order to exploit taxonomy classification together with free text searching, obtaining the power of a full- fledged text-retrieval system, Meta-search services (MetaCrawler (Selberg et al. 1995), WebCompass (Quarterdeck 1997), SavvySearch (Dreilinger 1997) and others) have been built that are able to use powerful free-text indexes like Altavista (Altavista 1997) as subroutines, querying all available services in parallel and then aggregating the results. Although these search engines present a sophisticated query interface, the results they deliver are currently perceived by the user community as unsatisfactory.

- Structural representation of sites A considerable amount of research has been made on how to complement keyword-based searching with databasestyle support for querying the Web. Several projects addressed this problem, and three main WWW query languages have been proposed so far: Web3QL (Konopnicki et al. 1995), WebSQL (Mendelzon et al. 1996) and WebLog (Lakshmanan et al. 1996). The first two languages are modelled after standard SQL used for RDBMS, while the third retains the flavour of the Datalog language. However, all these three languages give only a small fraction of the power of the original query languages they are based on, since they explicitly refrain from semantics representation issues. Web3QL and 
WebSQL offer a standard relational representation of Web pages, such as Document (url, title, text, type, length), which can be easily constructed from HTML tagging. The user can present SQL-like queries to Web sites based on that relational representation. Content- related queries (for instance: Document.text = Italy) are mapped in free-text searches using a conventional search engine. In addition to similar query capabilities, Web3QL offers an elementary graph pattern search facility, allowing users to search for simple paths in the graph representing the navigational structure of a Web site. Finally, WebLog (and its enhancements, (Giannotti et al. 1997)) propose an O-O instance representation technique which leads to a powerful deductive query language, fully equipped with recursion; but again it lacks any representation of data semantics.

- Instance-based semantics representation Nowadays it is widely recognized that to effectively build Web-based services, developers must be able to impose impose some sort of semantic structure upon Web sites in order to support efficient information capture (Hamilton 1997). In fact, the subject of semantics representation for Web sites in currently actively investigated. A well-known technique for instance-based semantics representation is semantic tagging, i.e. the use of extended HTML tags to represent semantic information. The basic idea underlying this approach is that a new kind of HTML tags can be used to superimpose a representation of semantics (based, for instance, on standard entity-relationship technique) on the navigational structure of a Web site. Semantic tags can be used to refer to an entity the data stored in a Web page and to denote relationships as semantic links that are not meant to be followed in navigation only, but used for querying purposes. Several variations of the semantic tagging idea ((Kogan et al. 1997)) have been proposed by various researchers (a logic-programming approach is presented in (Loke et al. 1997)). Moreover, HTML standard committees (W3C 1997) seem to be considering its partial endorsement. However, no effective query support based on semantic tagging is yet available. Other approaches try to address the problem of Web indexing and querying in the more general framework of dealing with semi-structured data. For instance, the Tsimmis system (Garcia-Molina et al. 1997) proposes an OEM object model to represent semistructured information together with a powerful query language, Laurel. For each Web site, the user defines OEM classes to be used in its Tsimmis representation. Then, an extraction technique based on a textual filter is applied, initializing objects from Web pages data. Indeed, Tsimmis additional DataGuide facility allows to identify regularities in the extracted instance representation to produce a full-fledged site schema. We are currently exploring translation of DataGuide schemata into WG-log in order to add query capability to Tsimmis sites.

- Schema-based semantics representation 
With the partial exception of Tsimmis, all the approaches described above lack an explicit notion of schema. This may be due to the fact that, while the advantages of schema-aware query formulation are widely recognized in the database context, this technique has been considered unfeasible on the WWW because no schema information is normally associated to Web sites. However, this situation is evolving as an increasing number of sites, particularly on Intranets, are being designed using well specified design methodologies such as HDM (Garzotto et al. 1995), RMM (Isakowitz et al. 1995) $Y O O$ (Balasubramanian et al. 1995) and the like. When a methodology is used to design a Web site, some notion of site schema is present during the site design process. Indeed, many commercial authoring environments for Web sites already hint to the idea of some sort of navigational schema to be choosen by the user as the basis of automatic site generation. Moreover, several research prototypes of Web site generators based on hypermedia design languages, are becoming available Some of these tools even translate the site schema into a relational representation (Fraternali et al. 1997). A representation of semantics based on a standard relational schema is also used in the Araneus project (Atzeni et al. 1997) where Web site crawling is employed to induce schemata of Web pages. These fine grained page schemata are later to be combined into a site-wide schema, and a specialpurpose language, Ulixes is used to build relational views over it. Rsulting relational views can be queried using standard SQL language, or trasformed in autonomous Web sites using a second special-purpose language, Penelope. It is worth observing that the Araneus approach to schema induction requires semi-structured Web site data to be converted in relational tables to allow database-style querying. In WG-log, graph-based instance and schema representations are used for query, while Web site data remain in their original, semi-structured form.

\section{A GRAPH DESCRIPTION LANGUAGE FOR WEB SITES: THE DATA MODEL}

The use of design methodologies for hypermedia applications is currently well estabilished and widely employed to develop multimedia hypertextual applications. Besides allowing conventional or object-oriented design elements, such as E/R-like entities or OMT-like classes, nearly all modern hypermedia specification languages are associated to a presentation and navigation semantics, clearly indicating how entities are to be presented to the user. This approach is justified by the fact that no query support is generally offered to hypermedia products, whose fruition is based on free user navigation. In our opinion, an effective Description and Manipulation Language for Web sites should be able to complement the hypermedia model with database-like querying capabilities. In this Section we describe $W G$-log, a graph-oriented language support- 
ing representation of both data model and structural entities. Graphs have indeed been an integral part of the database design process, and ever more so after the introduction of object-oriented data models; moreover they are naturally connected to graphical interfaces. WG-log has its formal basis in the graph-oriented language G-log (Paredaens et al. 1995). G-log, being designed as an object database language, only includes two node types ( representing abstract and concrete objects) and one link type (representing logical relationships, generally aggregations); WG-log extends G-log by including some standard hypermedia design notations that allow for linking conceptual entities and relationships to navigational concepts such as WWW pages and links. As a result, WG-log schemata cleanly denote both logical and structural concepts. In addition, there is the possibility to specify some hypertext features like index pages or entry points, which are essentially related to the hypertext presentation style. In this section we informally present the data model and the language of WG-Log; more formal definitions and examples of G-Log can be found in (Paredaens et al. 1995, Paredaens et al. 1997). References can also be found in (Garzotto et al. 1995), where the HDM hypermedia design language is presented. In WG-Log, directed labeled graphs are used as the formalism to specify and represent Web site schemata, instances, views (also called access structures), and queries. The nodes of the graphs stand for objects and the edges indicate relationships between objects. In WG-log schemata, instances and queries we distinguish four kinds of nodes:

- slots (also called concrete nodes), depicted as ellipses, indicate objects with a representable value; instances of slots are strings, texts, pictures, sound tracks, numbers, movies or movie frames (depending on the desired granularity of representation);

- entities, depicted as rectangles, indicate abstract objects such as monuments, professors, or cities; note that an abstract object can be chosen to correspond to one or more Web pages, possibly linked to each other in different ways: it is for the designer to decide which level of granularity the schema is meant to convey;

- collections, represented by a rectangle containing a set of horizontal lines, indicate collections or aggregates of objects, generally of the two types above; an instance of such a node is the index of all painters in a certain gallery (in this case we say that the collection is homogeneous) ;

- entry points, depicted as triangles, represent the unique page that gives access to a portion of the site (or to an alternative view of the site), for instance the site home page. To each entry point type corresponds only one node in the site instance. It is worth noticing that entry points and collection nodes can be used in queries for creating new access structures for providing alternative presentations of Web portions.

We also distinguish four kinds of graph edges: 
- structural edges, representing navigational links between pages; such an edge may stand for the link between a collection node representing the painter index and the entity of type painter;

- logical edges, representing logical relationships between objects; such an edge might connect painters to their paintings. The presence of a logical relationship does not necessarily imply the presence of a navigational link between the two entities at the instance level;

- double edges, representing a navigational link coupled with a logical link; such an edge might connect a painter to his/her paintings, also indicating that there is a navigational link that allows paintings to be reached from their author;

- Is_a edges, representing the inheritance relationship between objects; such an edge might connect painters and artists (as their generalization). Note that Is_a edges are only allowed in a WG-log schema, while do not make any sense at the instance and query level.

As an example of use of these lexical elements, Fig. 5 shows the WG-log schema while Fig. 6 contains an istance, namely the experimental WWW site whose URL is http://romeo.sci.univr.it/vrtour. This WG-log description was easily obtained as a part of the design process of the site; an HTML page is shown in Fig. 7. It should be also noted that all entities in a schema are marked by a unique code that will be exploited during query execution.

\subsection{WG-log schemata}

A (site) schema contains information about the structure of the Web site. This includes the (types of) objects that are allowed in the Web site, how they can be related and what values they can take. Logical as well as navigational (structural) elements can be included into a site schema, thus allowing for flexibility in the choice of the level of detail. In fact, index and entry point nodes, mainly related to the hypertext presentation style, may be used by designers who want to emphasize the hypertextual aspects of their design, while such elements can be dispensed with by those designers who are more interested in the conceptual contents of the site schema. As far as node granularity is concerned, an entity can be chosen to represent one or more site pages. For instance in a University site an entity Professor in the schema might be mapped in the instance to one page or several pages for each professor; in the latter case, pages referring to the same professor may be differently linked to each other, the only constraint on different implementations being the availability at query time of the information about instance references to their schema entities. Formally, a schema contains the following elements: 

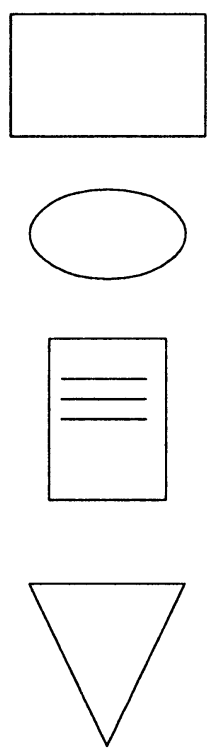

Relation Name
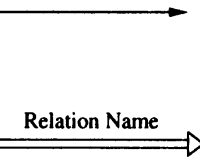

Isa

\author{
Entity
}

Slot (attribute)

\author{
Index
}

Entry-Point

Logical Link

Navigational Link
Coupled
Logical-Navigational
Link

"Is_a" Link

Figure 4 WG-log lexicon

- a set $S L$ of concrete object (or Slot) Labels,

- a set ENL of ENtity Labels, containing the special label dummy,

- a set COL of COllection Labels,

- a set LEL of Logical Edge Labels,

- one Structural Edge Label SEL (which in practice is omitted),

- a set DEL of Double Edge Labels,

- one Is_a edge label ISA,

- and a set $\mathcal{P}$ of productions*.

${ }^{*}$ We require that the productions form a set because we do not allow duplicate productions in a schema. 


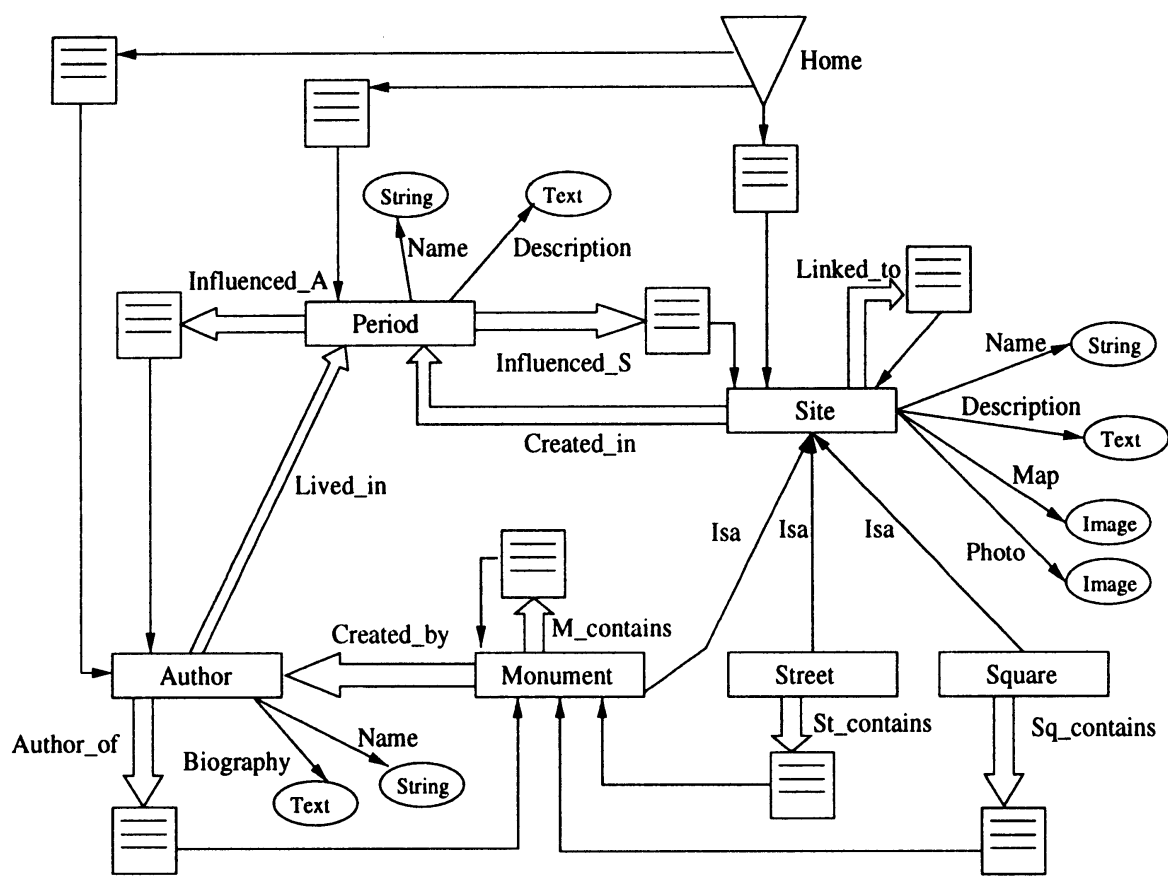

Figure 5 The WG-log schema

The productions dictate the structure of WG-Log instances (which are the actual sites); the productions are triples representing the types of the edges in the instance graph. The first component of a production always belongs to $E N L-\{d u m m y\} \cup C O L \cup E P$, since only non-concrete objects can be related to other objects. The second component is an edge label and the third component is an object label of any type. If the edge label is $I S A$, the two node labels must both belong to $E N L$. A Web site schema can be represented as a directed labelled graph, by taking all the objects as nodes and all the productions as edges. Note that two nodes might be connected by more than one edge. If multiple logical edges connect two nodes, they represent different relationships between those objects; the presence of a structural edge between two nodes represents the (possible) presence of a navigational direct link between the corresponding pages in the site instance: no two nodes, however, can be connected by more than one structural edge or by more than one ISA edge, since this would be meaningless. Finally, we assume a function $\pi$ associating to each slot label a set of constants, which is its domain; for instance, the domain of a slot of type image might be the set of all jpeg files. 


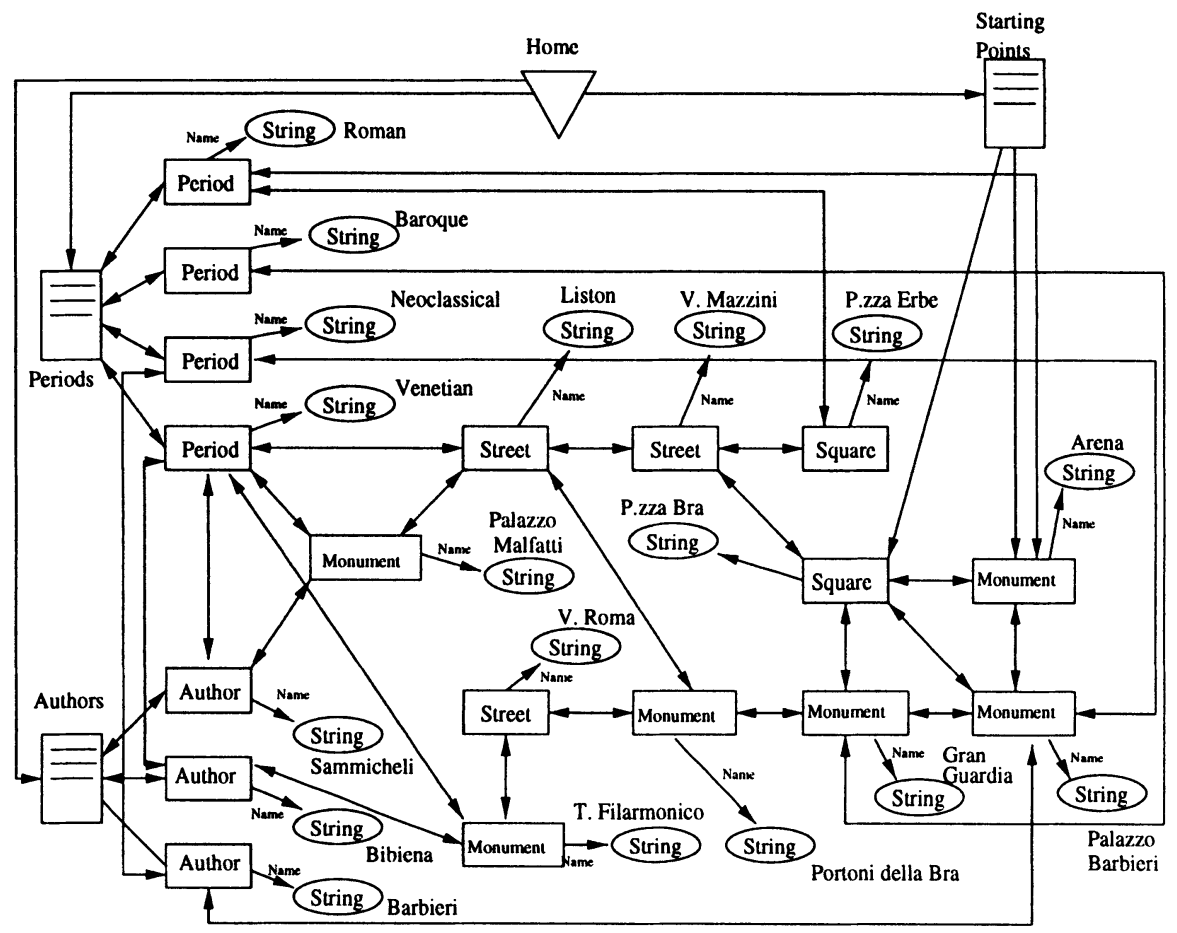

Figure 6 The site instance

\subsection{WG-log instances}

A (Web site) instance over a schema $\mathcal{S}$ contains the actual information that is stored in the Web site pages. It is a directed labeled graph $I=(N, E) . N$ is a set of labeled nodes. Each node represents an object whose type is specified by its label. The label $\lambda(n)$ of a node $n$ of $N$ belongs to $E P \cup S L \cup E N L-$ $\{d u m m y\} \cup C O L^{*}$. If $\lambda(n)$ is in $E P$, then $n$ is an entry point node, and it is the only one with label $\lambda(n)$; if $\lambda(n)$ is in $S L$, then $n$ is a concrete node (or a slot); if $\lambda(n)$ is in $E N L$, then $n$ is an abstract object, that can coincide with one or more or a part of site page; otherwise $n$ is a collection node, which means that it contains an aggregation of homogeneous or eterogeneous objects. If $n$ is concrete, it has an additional label print $(n)$, called the print label, which must be a constant in $\pi(\lambda(n))$. $E$ is a set of directed labeled edges. An edge $e$ of $E$ going from node $n$ to $n^{\prime}$ is denoted $\left(n, \alpha, n^{\prime}\right) . \alpha$ is the label of $e$ and belongs to $L E L \cup\{S E L\} \cup D E L^{*}$. The edges must also conform to the productions of the schema, so $\left(\lambda(n), \alpha, \lambda\left(n^{\prime}\right)\right)$ must belong to $\mathcal{P}$. Besides these edges, we also assume an implicit equality edge (a logical edge with an

* Note that no ISA edge is allowed in instances. 


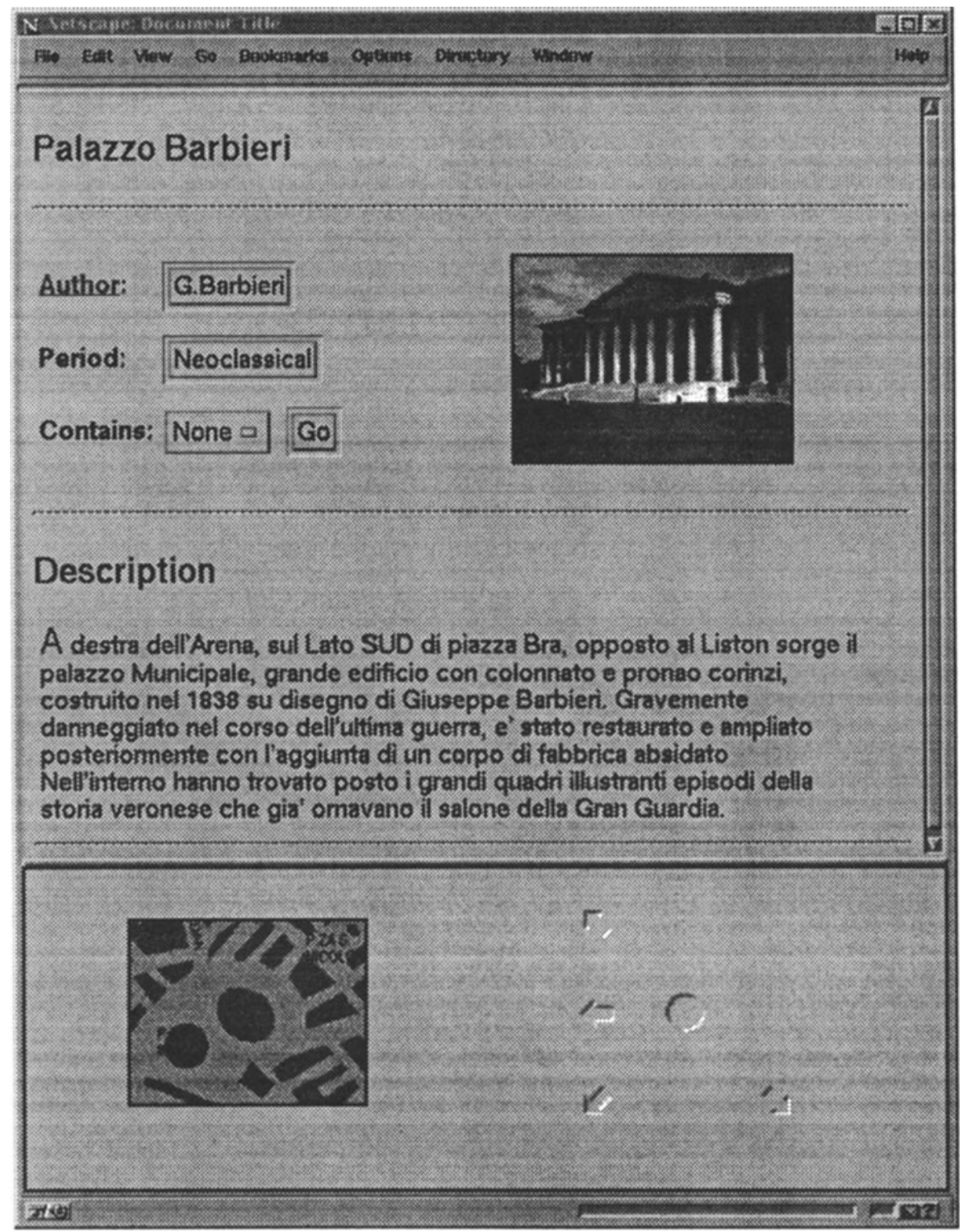

Figure 7 A page of our trial site 


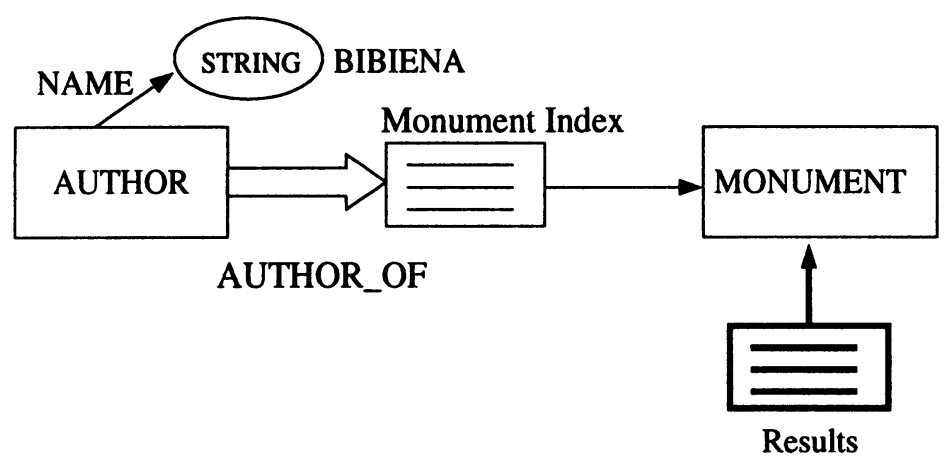

Figure 8 A WG-Log solid rule.

equality sign as label) going from each node of the instance to itself. Figure 6 contains an instance over the schema of Figure 5.

\section{WG-LOG RULES AND PROGRAMS}

A WG-Log query is a (set of) graph(s) whose nodes can belong to all four node types used in schemata, and whose edges can be logical, double or structural. This allows for pure database-like and pure navigational queries; w.r.t. our experimental site, a query could select all the monuments which are the work of a given author as opposed to another listing all the pages in the site linked to more than five distincts nodes. It is interesting to remark that this technique also opens the interesting possibility of mixed queries, e.g. listing the works index page of all authors. In all three cases, in fact, the query results in a transformation performed on the instance. WG-Log rules, programs and goals can be used to deduce, query and restructure information from the information contained in the Web site pages. Rules are themselves graphs, which can be arranged in programs in such a way that new views (or perspectives) of (parts of) the Web site be available for the user. Like Horn clauses, rules in WG-Log represent implications. To distinguish the body of the rule from the head in the graph $P$ representing the rule, the part of $P$ that corresponds to the body is colored red, and the part that corresponds to the head is green. Since this paper is in black and white, we use thin lines for red nodes and edges and thick lines for green ones. Figure 8 contains a WG-Log rule over the Web site schema of Figure 5. It expresses the query: Find all the monuments whose author is Bibiena. Note the use of the Result collection node in the rule: it will build an access structure in the resulting instance. The application of a rule $r$ to a site instance $I$ produces a minimal superinstance of $I$ that satisfies $r$. We say that an instance satisfies a rule if every matching of the red part of the rule in the instance can be extended to a matching of whole rule in the instance. The matchings of (parts of) rules in instances are called embeddings. For example, the instance $I$ of Figure 6 does not satisfy the rule $r$ of Figure 8. In fact, there 


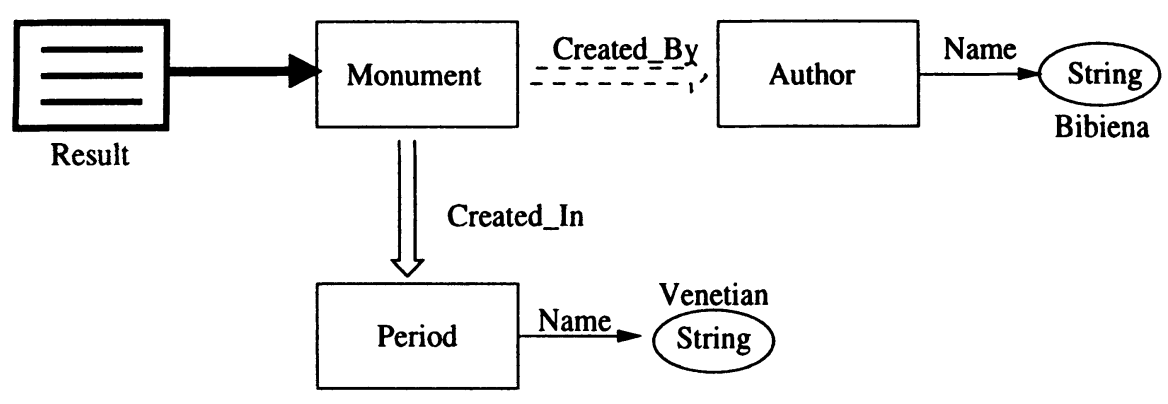

Figure 9 A WG-Log rule involving negation.

is one possible embedding $i$ of the red part of $r$ in $I$, hence, the monument -nodes pertaining to Bibiena of $I$ must be connected to a Result-node and this is not the case. Because $I$ does not satisfy $r, I$ is extended in a minimal way such that it satisfies $r$. In this case, the effect of rule application is that a Result-node is created and is linked to all the appropriate monumentnodes by an SEL -edge. Now the instance satisfies the rule, and no smaller superinstance of $I$ does, so this is the result of the query specified by the rule. Note that the new instance, obtained from the query, contains a new access structure (the node RESULT and its links to all Bibiena's monuments), which allows the retrieval of the nodes in an alternative way, that was not possible in the initial instance. We will also see in the sequel that WG-log also allows the expression of goals, in order to filter out non-interesting information from the instance obtained from the query. Rules in WG-Log can also contain negation in the body; we use solid lines to represent positive information and dashed lines to represent negative information. So a WG-Log rule can contain three colors: red solid (RS), red dashed (RD), and green solid (GS). The rule of Figure 9 expresses the query find all monuments of the venetian period whose author is not Bibiena. The instance $I$ of Figure 6 also does not satisfy this rule. The two possible embeddings of the RS part of $r$ in $I$ are applicable since they cannot be extended to embeddings of the RS and the RD part of $r$ in $I$. Hence, the monument-nodes of $I$ should be connected to a Result-node (to extend the found embeddings to embedding also of the GS part of $r$ in $I$ ), and this is not the case in $I$.

\subsection{WG-Log Rules and Goals}

We now formally define what WG-Log rules are and when an instance satisfies such a rule, or a set of such rules. As in G-log, WG-Log rules are constructed from patterns. A pattern over a Web site schema is similar to an instance over that schema. There are three differences: 1 ) in a pattern equality edges may occur between different nodes, having the same label, 2) in a pattern concrete nodes may have no print label, and 3) a pattern may contain entity nodes 
with the dummy label, used to refer to a generic instance node. A pattern denotes a graph that has to be embedded in an instance, i.e. matched to a part of that instance. An equality edge between two different nodes indicates that they must be mapped to the same node of an instance. A colored pattern over a schema is a pattern of which every node and edge is assigned one of the colors RS, RD, or GS. If $P$ is a colored pattern, we indicate by $P_{R S}$ the red solid part of $P$, by $P_{R S, R D}$ the whole red part of $P$, and by $P_{R S, G S}$ the solid part of $P$. In a generic colored pattern, these parts will not be patterns themselves, since they can contain dangling edges. However, for $P$ to be a WG-Log rule, we require that these subparts of $P$ be patterns. Formally, a $W G$ - Log rule $r$ consists of two schemata $\mathcal{S}_{1}$ and $\mathcal{S}_{2}$, and a graph $P . \mathcal{S}_{1}$ is called the source (schema) of $r$. $\mathcal{S}_{2}$ is a superschema* of $\mathcal{S}_{1}$, and is referred to as the target (schema) of $r . P$ must be a colored pattern over $\mathcal{S}_{2}$ such that $P_{R S}, P_{R S, R D}$ and $P_{R S, G S}$ are patterns over $\mathcal{S}_{2}$. Figure 8 contains the colored pattern $P$ of a rule, that has as source the schema of Figure 5, and as target the same schema, to which a Result-node and an in-edge are added. To define when an instance satisfies a rule, we need the notion of embedding. An embedding $i$ of a pattern $P=\left(N_{P}, E_{P}\right)$ in an instance $I=\left(N_{I}, E_{I}\right)$ is a total mapping $i: N_{P} \rightarrow N_{I}$, such that for every node $n$ in $N_{P}$ holds that

- either $\lambda(i(n))=\lambda(n)$ or

- There is a production $(\lambda(n), I S A, \lambda(i(n)))$ in the target scheme;

moreover, if $n$ has a print label, then $\operatorname{print}(i(n))=\operatorname{print}(n)$. Also, if $\left(n, \alpha, n^{\prime}\right)$ is an edge in $E_{P}$, then $\left(i(n), \alpha, i\left(n^{\prime}\right)\right)$ must be an edge in $E_{I}$. Let $P=(N, E)$ be a subpattern of the pattern $P^{\prime}$ and let $I$ be an instance. An embedding $j$ of $P^{\prime}$ in $I$ is an extension of an embedding $i$ of $P$ in $I$ if $i=j \mid N$. An embedding $i$ of $P$ in $I$ is constrained by $P^{\prime}$ if $P^{\prime}$ equals $P$ or if there is no possible extension of $i$ to an embedding of $P^{\prime}$ in $I$. We use the notion of "constrained" to express negation: an embedding is constrained by a pattern if it cannot be extended to an embedding of that pattern. Let $r$ be a WG-Log rule with colored pattern $P$ and target $\mathcal{S}_{2}$. An instance $I$ over $\mathcal{S}_{2}$ satisfies $r$ if every embedding $P_{R S}$ in $I$ that is constrained by $P_{R S, R D}$, can be extended to an embedding $P_{R S, G S}$ in $I$. As we informally mentioned before, the instance of Figure 6 does not satisfy the rule of Figure 9. The only embedding $i$ of $P_{R S}$ in $I$ is constrained by $P_{R S, R D}$ (because it cannot be extended to an embedding of $P_{R S, R D}$ in $I$ ), and cannot be extended to an embedding of $P_{R S, G S}$ in $I$. To express complex queries in WG-Log, we can combine several rules that have the same source $\mathcal{S}_{1}$ and target $\mathcal{S}_{2}$ in one WG-Log set. So, a WG-Log set $A$ is a finite set of WGLog rules that work on the same schemata. $\mathcal{S}_{1}$ is called the source (schema) of $A$ and $\mathcal{S}_{2}$ is its target (schema). The generalization of satisfaction to the case of WG-Log rule sets is straightforward. Let $A$ be a WG-Log set with target

${ }^{*}$ Sub- and superschema, sub- and superinstance, and sub- and superpattern are defined with respect to set inclusion. 
$\mathcal{S}$. An instance $I$ over $\mathcal{S}$ satisfies $A$ if $I$ satisfies every rule of $A$. In WG-Log is also possible to use goals. A goal over a schema $\mathcal{S}$ is a subschema of $\mathcal{S}$, and is used to select information of the Web site. Normally, a goal is combined with a query to remove uninteresting information from the resulting instance. The effect of applying a goal $G$ over a schema $S$ to an instance $I$ over $S$ is called $I$ restricted to $G$ (notation: $I \mid G$ ) and is the maximal subinstance of $I$ that is an instance over $G$. The definition of satisfaction of a WG-Log set is easily extended to sets with goals. If $A$ is a WG-Log set with target $\mathcal{S}_{2}$, then an instance $I$ over $G$ satisfies $A$ with goal $G$ if there exists an instance $I^{\prime}$ over $\mathcal{S}_{2}$ such that $I^{\prime}$ satisfies $A$ and $I^{\prime} \mid G=I$.

\subsection{WG-Log Programs and Semantics}

There is a strong connection between G-Log and first order predicate calculus. In (Paredaens et al. 1995) it is shown that for every formula on a binary many sorted first order language there is an effective procedure that transforms it into an "equivalent" set of G-Log rules and a goal; the converse is trivially true. Hence, G-Log can be seen as a graphical counterpart of logic. WG-log is only a syntactic variant of G-log, whose semantics we want to retain in order to keep its expressive power and representation capability; thus the same correspondence holds for WG-log. Consider for instance the rule of Figure 9. This may be expressed in First Order Logic as follows:

$$
\begin{aligned}
& \forall m \forall p \forall a \exists \text { result }:\{\text { created_in }(m, p) \wedge \operatorname{period}(p, " V \text { enetian" }) \wedge \\
& \wedge n a m e(a, \text { “Bibiena" }) \wedge \neg \text { created_by }(m, a)\} \Rightarrow S E L(\text { result }, m)
\end{aligned}
$$

Note that simpler languages like Datalog do not capture the whole expressive power of G-log: a Datalog rule is expressed in G-log by a simple rule containing red solid nodes and edges, and only one green edge. Thus, it is not possible to express the semantics of WG-log by translating it in Datalog. In the previous section we defined when an instance satisfies a WG-Log rule set; by examining the logical counterpart of WG-log, we get an intuition of the meaning of a WG-log rule; however, in order to use WG-Log as a query language we need to define its effect, i.e. the way it acts on instances to produce other instances; only in this way we will be able to isolate, among the infinity of instances that satisfy a certain rule, the one we choose as the rule's result. The semantics of a WG-Log set $A$ with source $\mathcal{S}_{1}$ and target $\mathcal{S}_{2}$ is thus a binary relation over instances defined by:

$$
\begin{aligned}
& \operatorname{Sem}(A)=\left\{(I, J) \mid 1 . I \text { is an instance over } \mathcal{S}_{1} \text { and } J \text { is an instance over } \mathcal{S}_{2}\right. \text {, } \\
& \text { 2. } J \text { satisfies } A \text {, } \\
& \text { 3. } J \mid \mathcal{S}_{1}=I \text {, } \\
& \text { 4. No subinstance of } J \text { satisfies conditions 1. to } 3 \text {. }
\end{aligned}
$$


Item 3 expresses the requirement that in WG-Log we only allow queries, and no updates. If a WG-Log rule contains a red dashed and a green solid part, then it can be satisfied either by adding the red dashed part to an instance or by adding the green solid part. Because of item 3, the source schema can be chosen is such a way that only one (or even none) of the two extensions is allowed. In this way the semantics of the rule also depends on its source schema. Item 4 expresses minimality. In general there will be more than one minimal result of applying a WG-Log set to an instance, which corresponds to the fact that WG-Log is non-deterministic and Sem is a relation and not a function. In WG-Log, it is allowed to sequence sets of rules. A WG-Log program $P$ is a finite list of WG-Log sets such that the target schema of each set of $P$ equals the source schema of the next set in the program. The source schema of the first set is the source (schema) of $P$, and the target schema of the last set is the target (schema) of $P$. The semantics $\operatorname{Sem}(P)$ of a WG-Log program $P=\left\langle A_{1}, \ldots, A_{n}\right\rangle$ is the set of pairs of instances $\left(I_{1}, I_{n+1}\right)$, such that there is a chain of instances $I_{2}, \ldots, I_{n}$ for which $\left(I_{j}, I_{j+1}\right)$ belongs to $\operatorname{Sem}\left(A_{j}\right)$, for all $j$. If a number of WG-Log rules are put in sequence instead of in one set, then, because minimization is applied after each rule, fewer minimal models are allowed. In fact, sequencing can be used to make a non-deterministic set of rules deterministic. Finally, a goal can be used in conjunction with a program. If $\mathcal{S}_{2}$ is the target of $P$ and $G$ is a goal over $\mathcal{S}_{2}$, then the semantics of $P$ with goal $G$ is: $\operatorname{Sem}(P, G)=\left\{(I, J) \mid \exists\left(I, J^{\prime}\right) \in \operatorname{Sem}(P)\right.$ such that $\left.J^{\prime} \mid G=J\right\}$. There are 3 complexity levels of constructions to express queries in WG-Log: rules, sets and programs, which all three can be used in conjunction with a goal. This results in the six cases stated in the table of Figure 10. The use of all the three complexity levels guarantees that WG-log is computationally complete (Paredaens et al. 1995), i.e., it can produce any desired superinstance of a given instance. Normally, one or two rules, together with a goal, are

\begin{tabular}{ccc}
\hline & without goal & with goal \\
\hline rule & WG-Log rule & WG-Log rule + goal \\
set of rules & WG-Log set & WG-Log set + goal \\
sequence of sets of rules & WG-Log program & WG-Log program + goal \\
\hline
\end{tabular}

Figure 10 The complexity levels of WG-Log queries.

sufficient to express most of the interesting queries we can pose to a Web site; however, some important queries do require the full language complexity. As an example, suppose we want to find all the pairs of nodes that are unreachable from each other by navigation; in other words, we want all the pairs that are not in the transitive closure of the relationship expressed by label $S E L$. An easy and natural way to solve this query is to compute the transitive closure 


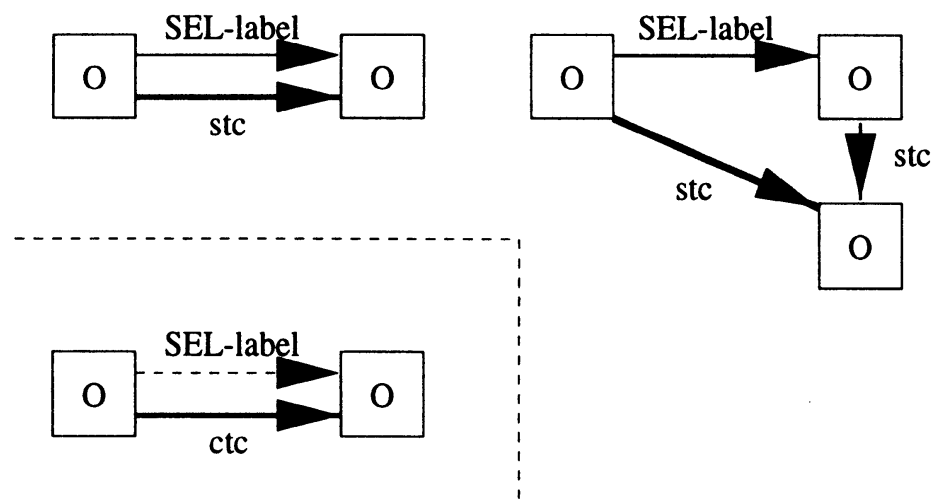

Figure 11 The complement of the navigational transitive closure.

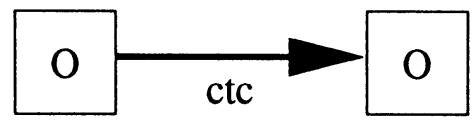

Figure 12 A Goal on the complement of the navigational transitive closure.

stc of $S E L$, and then take the complement $c t c$ of that relation. The WG-Log program of Figure 11 solves this problem. It is a sequence of two sets of rules. The first set, which consists of two rules, adds stc-edges (logical) between all nodes that are linked by a $S E L$-path. The second set has only one rule and takes the complement of the transitive closure by adding a ctc-edge if there is no stc-edge. Eventually, a goal can be added to select only the node pairs that are linked by the ctc (logical) relationship.

Another interesting query might ask all the nodes that are not reachable from a specific one, for instance the page of the artist Bibiena; in this case, the program must be complemented by the goal of Figure 12. Note typically, such goals can be used to optimize computation; however, this is outside the scope of this paper.

\section{EVALUATION OF WG-LOG PROGRAMS}

In order to be able to express a rich set of queries, we have conceived WG$\log$ as a language with a complex semantics; this gives rise to a computation algorithm that, in the general case, is very inefficient. However, in most cases the queries are expressed by only one or two rules, and possibly a goal which contributes to improving the efficiency of program computation. In the first subsection we present the computation algorithm in its most general form; later, we present an example of query computation, based on very simple 
data structures, which gives the flavour of the real complexity the system will have to tolerate without any improvements. In future work we will study appropriate data structures, and optimizations based on the goal structure, which will offer the possibility of increasing the efficiency of the naive approach presented here.

\subsection{A general Computation Algorithm}

We now present the FastComp algorithm, that computes the result of a generic WG-Log set by using a kind of backtracking fixpoint technique. Suppose we are given a set of rules $A=\left\{r_{1}, \ldots, r_{k}\right\}$ with source $\mathcal{S}_{1}$ and target $\mathcal{S}_{2}$, and a finite instance $I$ over $\mathcal{S}_{1}$. The procedure FastComp will try to extend $I$ to an instance $J$, in such a way that $J$ is finite and $(I, J) \in \operatorname{Sem}(A)$. If this is impossible, it will print the message: "No solution". FastComp calls the function Extend, which recursively adds elements to $J$ until $J$ satisfies $A$, or until $J$ cannot be extended anymore to satisfy $A$. In this last case, the function backtracks to points where it made a choice among a number of minimal extensions and continues with the next possible minimal choice. If the function backtracks to its first call, then there is no solution. In this sense, FastComp reminds the "backtracking fixpoint" procedure that computes stable models (Sacca et al. 1990).

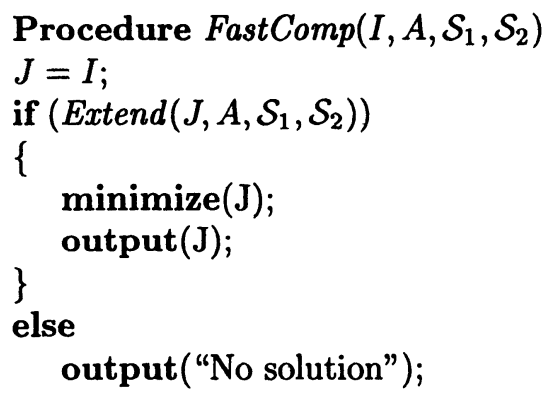

Function Extend $\left.\left(\operatorname{var} J, A, \mathcal{S}_{1}, \mathcal{S}_{2}\right)\right)$






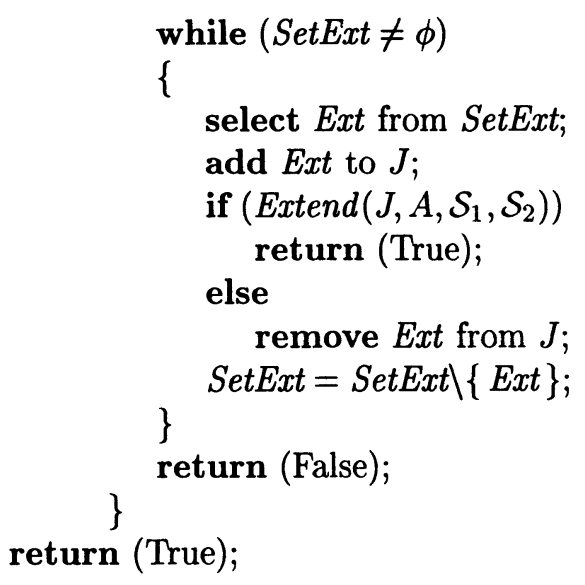

The algorithm uses the notion of "legal, minimal extension" of an instance. By legal, we mean that the extension may only contain nodes and edges not belonging to $\mathcal{S}_{1}$. Minimal indicates that no subpart of the extension is already sufficient to make the embedding under consideration extendible. We denote by $F$ ast $\operatorname{Comp}(A)$ the set of all the pairs of instances $(I, J)$, such that $J$ is an output of the FastComp algorithm, for inputs $I$ and $A$. In (Paredaens $e t$ al. 1997) we proved that the FastComp algorithm is sound and finitely complete:

FastComp $(A)=F \operatorname{Sem}(A)$, for every WG-Log set $A$. Note that the complexity of FastComp is accounted for by the high expressive power of the language. The algorithm reduces to the standard fixpoint computation for those WGLog programs that are the graphical counterpart of Datalog, i.e. sets of rules that consist of a red solid part and one green solid edge. Thus, efficiency of computation can easily be achieved for such programs, while optimization becomes more and more needed (and difficult) if more expressive queries are posed.

\subsection{An example of Rule Evaluation}

We shall now briefly comment on how FastComp can be used, at least in principle, to execute a WG-log query. Our sample query execution is based on three data structures:

- the (Typed) Adiacency Matrix TAM of the instance graph.

- the Instance Table IT linking schema entities and their instances

- the URL list $U L$ linking instances to HTML pages or other network objects.

The role of the instance table is in many respects similar to that of the ontology introduced in (Luke et al. 1997). Each entry of the adiacency matrix lists the 


\begin{tabular}{|c|c|c|c|c|c|c|c|c|c|c|c|c|c|c|c|c|c|c|c|}
\hline & 0 & 1 & 2 & 3 & 4 & 5 & 6 & 7 & 8 & 9 & 10 & 11 & 12 & 13 & 14 & 15 & 16 & 17 & 18 \\
\hline 0 & 0 & 1 & 1 & 1 & 1 & 1 & 1 & 1 & 0 & 0 & 0 & 0 & 0 & 0 & 0 & 1 & 0 & 1 & 0 \\
\hline 1 & 1 & 0 & 0 & 0 & 0 & 0 & 0 & 0 & 0 & 0 & 0 & 0 & 0 & 0 & 1 & 0 & 0 & 1 & \\
\hline 2 & 1 & 0 & 0 & 0 & 0 & 0 & 0 & 0 & 0 & 0 & 0 & 0 & 0 & 0 & 0 & 0 & 1 & 0 & \\
\hline 3 & 1 & 0 & 0 & 0 & 0 & 0 & 0 & 1 & 0 & 0 & 0 & 0 & 0 & 0 & 0 & 0 & 0 & 0 & \\
\hline 4 & 1 & 0 & 0 & 0 & 0 & 1 & 1 & 0 & 1 & 0 & 1 & 0 & 1 & 0 & 0 & 0 & 0 & 0 & \\
\hline 5 & 1 & 0 & 0 & 0 & 1 & 0 & 0 & 0 & 1 & 0 & 0 & 0 & 0 & 0 & 0 & 0 & 0 & 0 & \\
\hline 6 & 1 & 0 & 0 & 0 & 1 & 0 & 0 & 0 & 0 & 0 & $\bullet 1$ & 0 & 0 & 0 & 0 & 0 & 0 & 0 & \\
\hline 7 & 1 & 0 & 0 & 1 & 0 & 0 & 0 & 0 & 0 & 0 & 0 & 0 & 0 & 0 & 0 & 0 & 0 & 0 & \\
\hline 8 & 1 & 0 & 0 & 0 & 1 & 1 & 0 & 0 & 0 & 0 & 0 & 0 & 1 & 0 & 0 & 0 & 0 & 0 & \\
\hline 9 & 1 & 0 & 0 & 0 & 0 & 0 & 0 & 0 & 0 & 0 & 1 & 0 & 0 & 1 & 0 & 0 & 0 & 0 & \\
\hline 10 & 1 & 0 & 0 & 0 & 1 & 0 & 1 & 0 & 0 & 1 & 0 & 0 & 0 & 0 & 1 & 0 & 0 & 0 & \\
\hline 11 & 1 & 0 & 0 & 0 & 0 & 0 & 0 & 0 & 0 & 0 & 0 & 0 & 1 & 0 & 0 & 1 & 0 & 0 & \\
\hline 12 & 1 & 0 & 0 & 0 & 1 & 0 & 0 & 0 & 1 & 0 & 0 & 1 & 0 & 1 & 0 & 1 & 0 & 0 & \\
\hline 13 & 1 & 0 & 0 & 0 & 0 & 0 & 0 & 0 & 0 & 1 & 0 & 0 & 1 & 0 & 0 & 0 & 1 & 0 & \\
\hline 14 & 1 & 1 & 0 & 0 & 0 & 0 & 0 & 0 & 0 & 0 & 0 & 1 & 0 & 0 & 0 & 0 & 0 & 0 & \\
\hline 15 & 1 & 0 & 0 & 0 & 0 & 0 & 0 & 0 & 0 & 0 & 0 & 1 & 1 & 0 & 0 & 0 & 1 & 1 & \\
\hline 16 & 1 & 0 & 1 & 0 & 0 & 0 & 0 & 0 & 0 & 0 & 0 & 0 & 0 & 1 & 0 & 1 & 0 & 0 & \\
\hline 17 & 1 & 1 & 0 & 0 & 0 & 0 & 0 & 0 & 0 & 0 & 0 & 0 & 0 & 0 & 0 & 1 & 0 & 0 & \\
\hline 18 & 1 & 0 & 0 & 1 & 0 & 0 & 0 & 1 & 0 & 0 & 0 & 0 & 0 & 0 & 0 & 1 & 0 & 1 & \\
\hline
\end{tabular}

Figure 13 A simplified version of the adjacency matrix

typed links (navigational, logical or coupled) connecting a pair of nodes. For the sake of conciseness, Fig. 13 does not show such a matrix, but a simpler, binary matrix where each entry $i, j$ is 1 whenever instances $i$ and $j$ are linked by a navigational step, a logical relationships or both. Moreover, rows and columns pertaining to slots are not listed. Actually, we do not need to store slots in the TAM matrix; it is sufficient and surely less space-consuming to store them in auxiliary data structures pertaining to each single entity, in order to allow fast label matching. IT (Fig.14) associates the unique code of each schema entity to a list of unique numbers called instance identifiers; this allows the Query Manager to trace instances of schema-defined entities in the instance graph. Finally, the URL list associates each instance identifier to one or more HTTP URLs. This means that in our approach an istance of an entity is not necessarily a single page, though this will probably be the most frequent case. With respect to the sample query in Fig. 9, asking for the monuments of the Venetian period whose author is not Francesco Bibiena, we remark that the initial values of FastComp parameters are as follows: the whole instance of Fig. 6, the single rule of Fig. 9 and the source schema of Fig. 5. The target schema for this query can be easily deduced from the rule and will therefore be omitted.

To start with, IT is consulted to obtain the identifiers of the instance entities that match the rule entities. The following lists are obtained: Period $=\{1,2,3,4\}$, Monument $=\{8,10,13,16,17,18\}$. These lists are then used to 


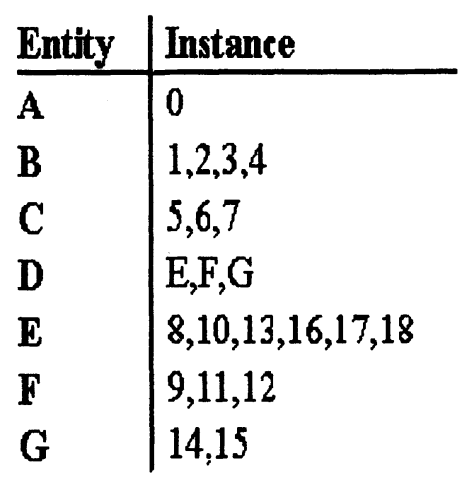

Figure 14 A sample instance table

extract from the TAM the following possible adiacency information:

$$
\begin{array}{ccc}
1 & 17 & 14 \\
2 & 16 & - \\
3 & 18 & - \\
4 & 8 & 10
\end{array}
$$

An equality test on the labels leaves only two possible embeddings for the red solid part of the rule: 4,8 and 4,10 . Now, we are ready to follow the tree of recursive calls of Extend for our sample FastComp execution. Luckily, the recursion depth turns out to be only four in this case.

first call of Extend

1st for iteration; embedding 4,8 does not satisfy rule

Red dashed valid extensions: 5 (from $T A M$ : the only instance of Venetian monument not related to Bibiena)

Green solid valid extensions: Result

SetExt $=\{5$, Result $\}$

1st while iteration

$J=J \cup\{5\}$

second call of Extend

1st for iteration; embedding 4,8 does not satisfy rule

Red dashed valid extensions: none

Green solid valid extensions: Result

SetExt $=\{$ Result $\}$

1st while iteration

$J=J \cup\{$ Result $\}$

3rd call of Extend

1st for iteration; embedding: 4,8 does satisfy rule

2nd for iteration; embedding: 4,10 does not satisfy rule 
Red dashed valid extensions: none

Green solid valid extensions: Result

SetExt $=\{$ Result $\}$

1st while iteration

$J=J \cup\{$ Result $\}$

4th call of Extend

1st for iteration; embedding: 4,8 satisfies rule

2nd for iteration; embedding: 4,10 satisfies rule

Returns True (ends 4th call)

Returns True (ends 3rd call)

Returns True (ends 2nd call)

Returns True (ends 1st call)

The instance thus obtained is minimal, thus it is a solution to the query.

\section{CONCLUDING REMARKS AND FUTURE WORK}

Experience with current WWW search engines has shown that the availability of a database-like schema is a prerequisite for any effective Web query mechanism. Though we are fully aware that the system described in this paper is only a preliminary step towards a satisfactory solution of the Web structuring and querying problem, we believe that its conceptual basis is sound and that its development may offer several interesting subjects for future research. For instance, a most important and promising issue is query execution itself, which must be both made more efficient and specialized to take into account the goal structure, schema information possibly available from a relational database underlying the site, and semantic properties of G-log, which enable the schema Robot to refuse a priori trivial or unsatisfiable queries. This is most needed since, as we have seen, $W G$-log retains the expressive power of the original $G$-log language: a carefully tuned execution mechanism is thus required to keep complexity in check (and to avoid "result explosion") when dealing with those queries that involve some kind of transitive closure. Another critical topic is the presentation of results: here not only efficiency considerations are involved, but also problems concerning the heterogeneous quality of the information stored: where text, images, sound tracks and similar pieces of information must be arranged to be shown to the user in a coherent and understandable way, architect's skills are needed, besides those of a Software designer. We plan to deal in the near future with querying federate Web sites. Namely, we plan to allow Web users to formulate queries on the basis of several site schemata at once, extending our query execution mechanism to take into account links between distinct Web sites. Finally, we plan to address at a later time more difficult problems like (semiautomatic) schema deduction on the basis of instance inspection; schema integration over unrelated sites; schema update at instance evolution; effective treatment of instance and schema graphs when these assume huge proportions. 


\section{Acknowledgments}

The authors wish to thank Riccardo Torlone for putting forward the idea that gave rise to the present research. Thanks are also due to Franca Garzotto for many useful discussions on the subject. We also like to thank the students Michele Baldi and Fabio Insaccanebbia, from the University of Verona, for their contribution to the design and development of the Schema Robot as a part of their Master's Thesis, and the anonymous referees of this paper for their careful reading and useful comments on an earlier version.

\section{REFERENCES}

Altavista, Inc. (1997) AltaVista Search Index http://www.altavista.digital.com.

Atzeni P., Mecca G. and Merialdo P. (1997) Semistructured and Structured Data in the Web: Going Back and Forth http://www.research.att.com/ suciu/workshop-papers.html.

Balasubramanian V., Bang Min M. and Joonhee (1995) M.Yoo: A Systematic Approach to Designing a WWW Application Communications of the ACM 38(8), 47-48.

Budi Y. and Dik Lun L. (1996) WISE: A World Wide Web Resource Database System IEEE Transanctions on Knowledge and Data Engineering 8(4), 548-554.

Damiani E. and Fugini M.G. (1995) Automatic Thesaurus Construction Supporting Fuzzy Retrieval of Reusable Software in Proceedings of ACM$S A C^{\prime} 95$, Nashville.

Dreilinger D. (1997) SavySearch Home Page http://www.lycos.com.

Fletcher J. (1990) Jumpstation FrontPage http://www.stir.ac.uk/js.

Fraternali P. and Paolini P. (1997) Autoweb: Automatic Generation of Web Applications from Declarative Specifications http://www.ing.unico.it/Autoweb.

Garcia-Molina H. and Hammer J. (1997) Integrating and Accessing Heterogeneous Information Sources in Tsimmis in Proceedings of ADBIS 97, St. Petersburg.

Garzotto F., Mainetti and L. Paolini P. (1995) Hypermedia Design Languages Evaluation Issues Communications of the ACM bf 38(8), 74-86.

Giannotti F., Manco G. and Pedreschi D. (1997) A Deductive Data Model for Representing and Querying Semistructured Data in Proceedings of the ILCP 97 Post-Conference Workshop on Logic Programming Tools for Internet Applications, Leuwen.

Gyssens M., Paredaens J., Van der Bussche J. and Van Gucht D. (1994) A Graph-oriented Object Database Model IEEE Transactions on Knowledge and Data Engineering, 6(4), 572-586.

S. Hamilton (1997) E-Commerce for the 21st Century IEEE Computer, 30(5), 44-47. 
Hu J., Nicholson D., Mungall C., Hillyard A. and Archibald A. (1996) WebinTool: A Generic Web to Database Interface Building Tool in Proceedings of the 1996 DEXA Workshop.

Isakowitz T., Stohr Edward A. D. and Balasubramanian, P. (1995) RMM: a Language for Structured Hypermedia Design Communications of the $A C M$, bf 38(8).

Kogan Y., Michaeli D., Sagiv Y. and Shmueli O. (1997)Utilizing the Multiple Facets of WWW Contents in Proceedings of the 1997 NGITS Workshop.

Konopnicki D. and Shmueli O. (1995) W3QL: A Query System for the World Wide Web in Proceedings of the 21th International Conference on Very Large Databases, Zurich.

Lakshmanan L., Sadri F. and Subramanian I. (1996) A Declarative Language for Querying and Restructuring the Web in Proceedings of the 1996 IEEE RIDE-NDS Workshop.

Loke S.W. and Davison A. (1997) LogicWeb: Enhancing the Web with Logic Programming http://www.cs.mu.oz.au/ ${ }^{\sim}$ swloke/papers/lw.ps.gz.

Lucarella D. and Zanzi A. (1996) A Visual Retrieval Environment for Hypermedia Information Systems ACM Transactions on Information Systems, 14(1).

Luke S., Spector L., Rager D. and Hendler J. (1997) Ontology-based Web Agents http://www.cs.umd.edu/projects/plus/SHOE.

Lycos, Inc. (1997) The Lycos Catatog of the Internet http://www.lycos.com.

Lycos, Inc. (1997) Point http://www.pointcom.com.

McBryan, O. A. (1994) WWWW and GENVL: Tools for Taming the Web, in Proceedings of the First Annual WWW Conference, Geneva.

The McKinley Group, Inc. (1997) Magellan Internet Guide http://www.cs.colostate.edu/dreiling/smartform.html/.

Mendelzon A., Mihaila G. and Milo T. (1996) Querying the World Wide Web, in Proceedings of the Conference on Parallel and Distributed Information Systems, Toronto.

Paredaens J., Peelman P. and Tanca L. (1995) G-log: A Graph-based Query Language IEEE Transactions on Knowledge and Data Engineering, (7), 436-453.

Paredaens J., Peelman P. and Tanca L. (1997) Merging Graph-Based and Rule-Based Computation, Data and Knowledge Engineering, to appear.

Pinkerton B. (1997) Finding What people Want: Experiences with WebCrawler, in Proceedings of the Second Annual WWW/Mosaic Conference, Geneva.

Quarterdeck Inc. (1997) Web Compass Fact Sheet http://www.arachnid.qdeck.com/qdeck/products/webcompass/.

Sacca D., Zaniolo C. (1990) Stable Models and Non-Determinism in Logic 
Programs with Negation in Proceedings of the 1990 PODS Conference. Selberg E. and Etzioni O. (1995) Multiservice Search and Comparison Using MetaCrawler in Proceedings of the Fourth International $W W W$ Conference.

Torlone R. (1996) Linguaggi di Interrogazione per il World Wide Web in Proceedings of SEBD '96, S. Miniato.

Yahoo, Inc.(1997) Yahoo! http://www.yahoo.com.

World Wide Web Consortium (1997) HTML 4.0 Specification Working Draft http://www.w3.org/TR/WD-html.

\section{BIOGRAPHIES}

Ernesto Damiani is an Assistant Professor at the University of Milan Research Centre located in Crema, Italy. He received the Laurea degree in Engineering from the University of Pavia and a Ph.D. in Computer Science from the University of Milan. His current research interests include semi-structured and structured information processing, soft computing, software reuse and object-oriented distributed computing, and has published a number of papers on these subjects. He is a member of the Steering Committee of the ACM Symposium on Applied Computing.

Letizia Tanca is currently a Full Professor at the University of Verona, and cooperates with Politecnico di Milano. She received her Laurea degree in Mathematics from the University of Naples, and her Ph.D. from the Politecnico di Milano. Her current research interests include advanced database systems, logic programming, and new paradigms for querying semistructured data. She is the author of several journal and conference papers on these subjects; she is also an author of the book "Logic Programming and Databases", published by Springer Verlag. 